\title{
EVAPOTRANSPIRAÇÃO REAL OBTIDA ATRAVÉS DA RELAÇÃO ENTRE O COEFICIENTE DUAL DE CULTURA DA FAO-56 E O NDVI
}

\author{
BERGSON GUEDES BEZERRA ${ }^{1}$, BERNARDO BARBOSA DA SILVA ${ }^{1}$, JOSÉ RENATO CORTEZ \\ BEZERRA $^{2}$, ZIANY NEIVA BRANDÃO ${ }^{2}$ \\ ${ }^{1}$ UNIVERSIDADE FEDERAL DE CAMPINA GRANDE, CAMPINA GRANDE, PB. \\ ${ }^{2}$ EMBRAPA - CENTRO NACIONAL DE PESQUISA DO ALGODÃO, CAMPINA GRANDE, PB.
}

\begin{abstract}
bergson.bezerra@gmail.com,bernardo@dca.ufcg.edu.br, renato@cnpa.embrapa.br, ziany_neiva@hotmail.com
\end{abstract}

Recebido Agosto 2009 - Aceito Dezembro 2009

\begin{abstract}
RESUMO
Um requisito fundamental para adoção de manejo da irrigação é a determinação diária da evapotranspiração (ET) das culturas. Em caráter operacional o método do coeficiente de cultura proposto pela Food Agriculture Organization (FAO), através do seu relatório 56 (Irrigation and Drainage Paper), é largamente utilizado na determinação da ET, e tem apresentado precisões que o tornam mundialmente aceito. A ET com base no coeficiente de cultura $(\mathrm{Kc})$, obtido a partir de índices de vegetação, particularmente o NDVI, tem sido calculada em vários estudos e para diversas culturas alcançando muita precisão, quando comparado com observações de campo. Diante do exposto, este trabalho teve por objetivo calcular a ET diária e sazonal da cultura do algodoeiro utilizando o método do Kc dual obtido em função do Índice de Vegetação por Diferença Normalizada - NDVI, obtido a partir de imagens TM - Landsat 5 livre da presença de nuvens. Os resultados revelaram precisões bastante confiáveis, pois foram verificadas diferenças menores que $10 \%$, quando comparados com valores da ET obtidos pela técnica da Bowen, corroborando com o desempenho alcançado pelo método em outras pesquisas realizadas em outras regiões do planeta. Dessa forma, pode-se concluir que o método apresenta bastante confiabilidade e simplicidade. Palavras-chave: Índice de Vegetação, Fluxo de Calor Latente, Razão de Bowen, Evapotranspiração de Referência. TM - Landsat 5.
\end{abstract}

ABSTRACT: REAL ACTUAL EVAPOTRANSPIRATION OBTAINED THROUGH THE RELATIONSHIP BETWEEN THE FAO-56 CROP DUAL COEFFICIENT AND NDVI

A fundamental requirement for adoption of irrigation management is to determine the crop daily evapotranspiration (ET). On an operational basis the crop coefficient method proposed by the Food and Agriculture Organization (FAO) through its report 56 (Irrigation and Drainage Paper) is widely used in the determination of ET and due to its accurate estimative, it has been globally accepted. The ET-based crop coefficient $(\mathrm{Kc})$ obtained from vegetation indices, particularly the Vegetation Index Normalized Difference (NDVI) has been measured in several studies and various crops showing great accuracy when compared to field observations. The objective of this study was to calculate the daily and seasonal ET of cotton crop using the method of dual Kc obtained as a function of the NDVI obtained from TM - Landsat 5 images clouds free. The obtained results are reliable, because less than $10 \%$ differences were found when comparing to the Bowen ratio technique observations, corroborating to the method performance achieved in other experiments conducted in other regions of the Earth. Thus, one can conclude that the method both simple and reliable.

Keywords: Vegetation Index, Latent Heat Flux, Bowen Ratio, Reference Crop Evapotranspiration. TM - Landsat 5. 


\section{INTRODUÇÃO}

Um requisito fundamental para adoção de manejo da irrigação, assim como do seu desempenho, é a determinação diária da evapotranspiração (ET) das culturas.

Em caráter operacional, a metodologia proposta pela Food Agriculture Organization (FAO) em seu boletim n ${ }^{\circ} 56$ (Allen et al., 1998) é largamente utilizado na determinação da ET, provavelmente pela sua relativa simplicidade, pois requer apenas o valor do coeficiente de cultura Kc,, além de informações meteorológicas (Doorenbos e Kassam, 1979; Allen et al., 1998). Essa metodologia tem apresentado níveis de precisão que o tornam mundialmente aceito (Hunsaker et al., 2003).

A abordagem da FAO-56 é baseada na combinação dos conceitos da evapotranspiração de referência (ET0) e do Kc, Equação 01, "simples" e/ou "dual" (Allen et al., 1998, Allen, 2000). O Kc "simples" combina os efeitos da evaporação do solo e da transpiração da cultura de forma única, enquanto que a metodologia do Kc "dual" consiste na conjunção do coeficiente basal da cultura (Kcb) e do coeficiente de evaporação do solo - Ke (Allen et al., 1998; Allen, 2000).

$$
K_{c}=\left(K_{e}+K_{b}\right)=E T / E T_{0}
$$

A ET0 representa a demanda atmosférica, enquanto que o coeficiente de cultura Kc varia predominantemente de acordo com as características específicas de cada cultura, com o estágio fenológico, com a umidade do solo e de uma forma limitada, com o tempo (Allen et al., 1998). Ainda de acordo com os mesmos autores o Kc representa basicamente a fração da ET presente na ET0, e constitui uma integração dos efeitos das principais características que distinguem uma cultura qualquer das culturas hipotéticas de referência (grama ou alfafa), que são altura, albedo, resistência estomática e a evaporação do solo.

Os procedimentos de cálculo do $\mathrm{K}_{\mathrm{e}}$ são fundamentados no balanço hídrico diário à camada superficial do solo, e requer como variáveis de entrada parâmetros físicos do solo, tais como capacidade de campo, ponto murcha permanente e água evaporável (Allen et al., 1998; Allen, 2000). Por outro lado, o cálculo do Kcb se faz necessário para os estágios fenológicos médio e final, e para condições climáticas especificadas no boletim FAO-56 (Allen et al., 1998). Para o cálculo do Kcb são requeridas medições da velocidade do vento a $2 \mathrm{~m}$ da superfície do solo, da média diária da umidade relativa do ar mínima e da altura das plantas.

Entretanto, tem sido demonstrado que a refletância espectral das culturas pode fornecer uma estimativa indireta do Kc. Essas estimativas são possíveis porque tanto o Kc, como a refletância espectral são sensíveis ao índice de área foliar e à fração de cobertura do solo (Choudhury et al., 1994, Moran et al., 1995). Similaridades entre o padrão sazonal do Kcb e os índices de vegetação para a cultura do trigo, foram apresentadas por Jackson et al. (1980), enquanto que Bausch e Neale (1987) e Neale et al. (1989) estabeleceram relações entre o Kcb e índices de vegetação para cultura do milho.

A ET com base no Kc obtido a partir de índices de vegetação, particularmente o NDVI, tem sido calculada em vários estudos e para diversas culturas (Hunsaker et al., 2003; Hunsaker et al., 2005a, b; Duchemin et al., 2006; Er-Raki et al., 2007; Hunsaker et al., 2007a, b; Simmoneaux et al., 2008; López-Urrea et al., 2009a). Nesses estudos o $\mathrm{K}_{\mathrm{e}}$ foi calculado com base no balanço hídrico diário conforme a metodologia da FAO-56 (Allen et al., 1998), porém Simmoneaux et al. (2008) calcularam o $\mathrm{K}_{\mathrm{e}}$ com base no NDVI. Em todos os estudos citados, as precisões alcançadas foram consideradas muito boas, quando comparadas com valores da ET observadas em campo oriundas dos mais variados métodos, apresentado diferenças não superiores a $10 \%$.

Diante do exposto, o presente estudo teve como objetivo estimar a evapotranspiração diária e sazonal da cultura do algodão, utilizando a relação entre o coeficiente de cultura dual proposta na FAO-56 e o NDVI obtido a partir de imagens digitais TM - Landsat 5, além de comparar os resultados obtidos com valores da ET estimados através da técnica de razão de Bowen.

\section{MATERIAL E MÉTODOS}

\section{1 Área Experimental}

A campanha experimental foi realizada na Base Física da EMPARN (Empresa de Pesquisa Agropecuária do Estado do Rio Grande do Norte) localizada sobre a Chapada do Apodi, no município de Apodi-RN (5037'37'S; 3749'54"W; e elevação de 138 m), cuja localização em relação ao Estado do Rio Grande do Norte e ao Brasil, está apresentada na Figura 1. O clima da região, de acordo com a classificação climática de Thornthwaite (1948), é do tipo DA'da', isto é, semi-árido, megatérmico, pequeno ou nenhum excesso de água e concentração da evapotranspiração potencial no trimestre mais quente (novembro, dezembro e janeiro). A precipitação media anual é de $920 \mathrm{~mm}$, com quadra mais chuvosa se estendendo de março a junho, conforme a figura 2. Os solos da área experimental, em sua maioria, são classificados como Cambissolo Eutrófico.

\subsection{Campanha Experimental}

A ET diária de um campo de 3,0 ha de algodão herbáceo (Gossipium hirsutum L., cultivar BRS 187 8H) irrigada por aspersão convencional foi estimada diariamente 


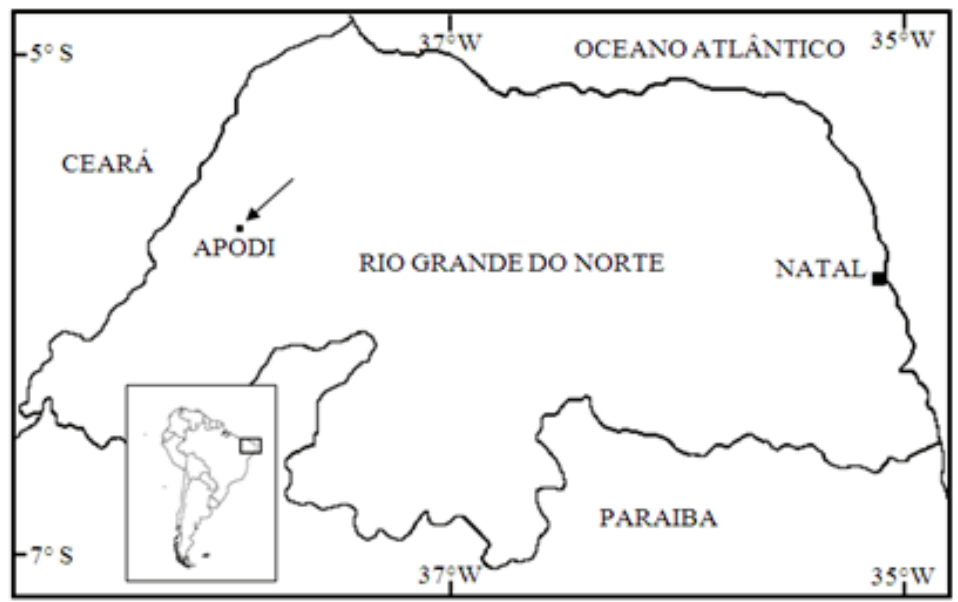

Figura 1 - Localização da área experimental em relação ao estado do Rio Grande do Norte e ao Brasil

entre o período de $04 / 10 / 2008$ a 12/01/2009, correspondendo a todo ciclo fenológico da cultura a partir do fluxo de calor latente - LE baseado na razão de Bowen, assumindo a igualdade entre os coeficientes de troca turbulenta de calor e vapor de água $(\beta=\mathrm{H} / L E \cong \gamma \Delta T / \Delta e)$ como segue:

$$
L E=\frac{R n-G}{1+\gamma \Delta T / \Delta e}
$$

onde: Rn é o saldo de radiação ( $\left.\mathrm{W} \mathrm{m}^{-2}\right), \mathrm{G}\left(\mathrm{W} \mathrm{m}^{-2}\right)$ é o fluxo de calor no solo $\gamma$ é a constante psicrométrica $\left(\mathrm{KPa}^{\circ} \mathrm{C}^{-1}\right), \Delta \mathrm{T}$ e $\Delta \mathrm{e}$ são os gradientes de temperatura $\left({ }^{\circ} \mathrm{C}\right)$ e pressão de vapor $(\mathrm{KPa})$, respectivamente, acima do dossel vegetativo. A ET

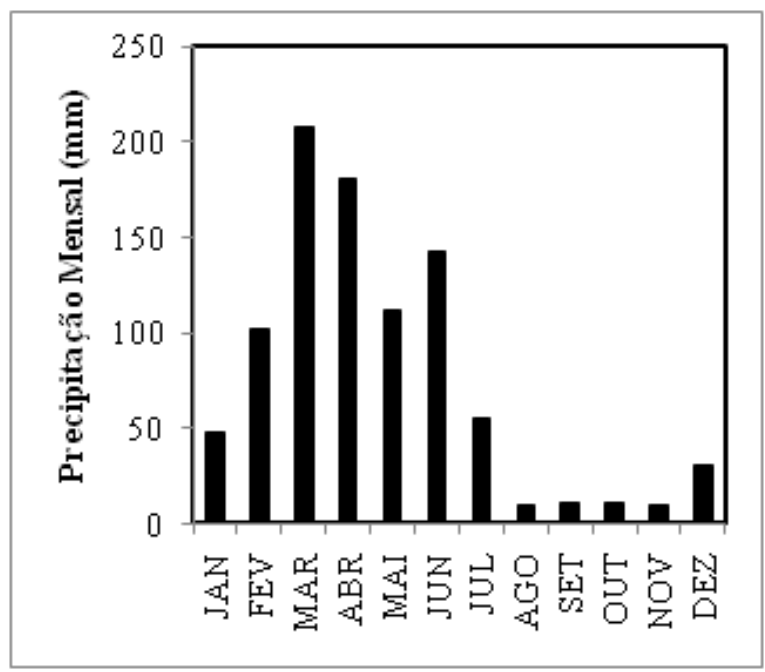

Figura 2 - Precipitação média mensal de Apodi-RN. Fonte: INMET da cultura do algodão foi calculada dividindo o valor d£2LE, Equação 02, pelo calor latente de vaporização da água. Seguindo proposta de Perez et al. (1999), apenas o período diurno, ou quando $(\mathrm{Rn}-\mathrm{G}>0)$ foi considerado.

As medições de Rn foram obtidas através de um saldo radiômetro NR-LITE (Campbell Sci., Logan-UT, USA), enquanto que $\mathrm{G}$ foi medido por duas placas HFP01SC-L Hukseflux Self-Calibrating Soil Heat Flux Plate (Campbell Sci., Logan-UT, USA) a 2,0 cm de profundidade. Já os gradientes de temperatura e pressão de vapor foram obtidos a partir das medidas das temperaturas seca e úmida através de psicrômetros construídos de termopares tipo $\mathrm{T}$ (cobre-constantan) a $0,5 \mathrm{~m}$ a 1,5 $\mathrm{m}$ acima do dossel. Todos os sensores empregados no cômputo do LE fizeram coletas a cada 5 segundos, armazenando as médias a cada 20 minutos em um sistema de aquisição de dados CR3000 (Campbell Sci., Logan-UT, USA). Dessa forma, a ET é calculada para cada 20 min e o valor diário foi obtido através da integração dos valores diurnos.

\subsection{Evapotranspiração de Referência}

A $\mathrm{ET}_{0}$ diária foi calculada mediante o método $\mathrm{FAO}$ Penman-Monteith (Allen et al., 1998), com base em dados meteorológicos coletados na estação meteorológica de

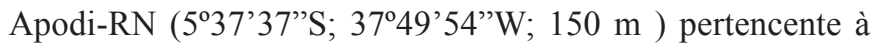
rede de estações automáticas do INMET - Instituto Nacional de Meteorologia, segundo expressão:

$$
E T_{0(24 h)}=\frac{0,408 \Delta(R n-G)+\gamma \frac{900}{T+273} u_{2}\left(e_{s}-e_{a}\right)}{\Delta+\gamma\left(1+0,34 u_{2}\right)}
$$


onde: T é a temperatura média diária do ar $\left[{ }^{\circ} \mathrm{C}\right] ; \mathrm{u}_{2}$ é a velocidade média diária do vento a $2 \mathrm{~m}$ de altura $\left(\mathrm{m} \mathrm{s}^{-1}\right)$, cujo valor foi previamente ajustado através da Equação 05 (Allen et al., 1998), pois as medidas disponíveis foram coletadas a uma altura de 2,0 m.; $\mathrm{e}_{\mathrm{s}}$ é a pressão de saturação $[\mathrm{kPa}] ; \mathrm{e}_{\mathrm{a}}$ é a pressão real de

$$
u_{2}=u_{10} \frac{4,87}{\ln (67,8 \cdot z-5,42)}
$$

vapor $[\mathrm{kPa}] ; \mathrm{e}_{\mathrm{s}}-\mathrm{e}_{\mathrm{a}}$ é o déficit de pressão de vapor $[\mathrm{kPa}] ; \Delta$ é a tangente à curva de pressão de vapor $\left[\mathrm{kPa}^{\circ} \mathrm{C}^{-1}\right]$.

onde: $\mathrm{u}_{10}$ é a velocidade do vento coletada a $10 \mathrm{~m}$ de altura e $\mathrm{z}$ é a altura $(10 \mathrm{~m})$.

\section{4 - Método de cálculo do Kc}

O NDVI foi calculado a partir de três imagens TM Landsat 5 livres da presença de nuvens adquiridas gratuitamente junto ao INPE - Instituto Nacional de Pesquisa Espaciais durante o período experimental, nos dias 01 e 17/11/2008 além de 19/12/2008 da órbita/ponto 216/64. Para o cálculo da ET foi utilizado o método do $\mathrm{K}_{\mathrm{c}}$ dual proposto no boletim FAO-56 (Allen et al., 1998), conforme equação seguinte:

$$
E T=\left(K_{c b}+K_{e}\right) \cdot E T_{0}
$$

onde: $\mathrm{K}_{\mathrm{cb}}$ é o coeficiente basal da cultura e $\mathrm{K}_{\mathrm{e}}$ é o coeficiente de evaporação do solo.

$\mathrm{O} \mathrm{K} \mathrm{cb}_{\mathrm{c}}$ foi obtido a partir da relação linear entre NDVI e $\mathrm{K}_{\mathrm{cb}}$ segundo relação aplicada por Simonneaux et al. (2008):

$$
K_{c b}=1,64 \cdot\left(N D V I-N D V I_{\min }\right)
$$

onde: NDVI é o índice de vegetação da cultura do dia estudado e NDVI $I_{\min }$ é o valor do NDVI para solo exposto, sendo que foi adotado o valor de 0,15 , corresponde ao valor médio para solo exposto obtidos com base nas três imagens.

A primeira etapa para obtenção do NDVI consistiu na conversão do Número Digital - ND das imagens TM - Landsat 5 em radiância espectral $\mathrm{L}_{\lambda}$, através da Equação 07 (Markham e Baker, 1986):

$$
L_{\lambda}=a_{b}+\left(\frac{b_{\lambda}-a_{\lambda}}{255}\right) \cdot N D
$$

onde: $a_{\lambda}$ e $b_{\lambda}$ são os coeficientes de calibração espectral do sensor (Chander e Markham, 2003).

A etapa seguinte compreendeu o cômputo das refletâncias das bandas, respectivamente do vermelho e infravermelho próximo, 3 e 4 do $\mathrm{TM}$ - Landsat $5-\rho_{\lambda}$, segundo a equação seguinte:

$$
\rho_{\lambda}=\frac{\pi \cdot L_{\lambda}}{E S U N_{\lambda} \cdot \cos \theta \cdot d r}
$$

em que: $E_{S U N}$ é a constante solar espectral das bandas 3 e 4 do TM - Landsat 5 segundo Markham e Barker, (1986), $\cos \theta$ é o co-seno do ângulo de incidência dos raios solares a normal da superfície e $\mathrm{d}_{\mathrm{r}}$ é o inverso do quadrado da distância relativa Terra-Sol, calculada em função do Dia Seqüencial do Ano DSA, pela equação seguinte:

$$
d r=1+0,033 \cdot \cos \left(D S A \frac{2 \pi}{365}\right)
$$

Finalmente, de posse das refletâncias das bandas 3 e 4, calculou-se o NDVI (Rouse et al., 1974):

$$
N D V I=\frac{\rho_{4}-\rho_{3}}{\rho_{4}+\rho_{3}}
$$

$\mathrm{O} \mathrm{K}_{\mathrm{e}}$ foi calculado com base na equação seguinte (Simmoneaux et al., 2008):

$$
K_{e}=(1-f \mathcal{C}) \cdot K_{e, \max }
$$

onde: fc é a fração de cobertura da vegetação: $f c=1,18$ (NDVI - NDVI ${ }_{\min }$ ) (Simonneaux et al., 2008) e $\mathrm{K}_{\mathrm{e}, \text { max }}$ representa o máximo coeficiente de evaporação do solo, ou seja, a máxima taxa de evaporação, que ocorre em períodos que sucedem eventos de precipitação ou irrigação que foi calculado para cada dia estudado através da equação seguinte (Allen et al., 1998), com as considerações descritas na seqüência:

$$
K_{e} \leq f_{e w} \cdot K_{c, \text { max }} \Rightarrow K_{e, \text { max }}=f_{e w} \cdot K_{c \text { max }}
$$

onde: $\mathrm{K}_{\mathrm{c}, \max }$ representa o limite superior da evaporação e da transpiração de qualquer superfície cultivada (Allen et al., 1998) calculado pela Equação 14 e $\mathrm{f}_{\mathrm{ew}}$ é a fração de solo úmido e exposto, que foi calculado pela equação seguinte:

$$
f_{e w}=\min \left(1-f c, f_{w}\right)
$$

onde: min indica que a escolha deve recair no menor valor dos termos da equação, $\mathrm{e}_{\mathrm{w}}$ é a fração do solo úmido por precipitação e irrigação. 
Para o cálculo do $\mathrm{K}_{\mathrm{e}, \max }$ foi admitido uma situação em que $\mathrm{f}_{\mathrm{w}}$ é máximo utilizando o valor proposto na Tabela 20 do boletim FAO-56 (Allen et al., 1998), para solo úmido irrigado por aspersão:

$$
\begin{aligned}
K_{c, \text { max }}= & \max \left(\left\{1,2+\left[0,04\left(u_{2}-2\right)-,\right.\right.\right. \\
& \left.\left.\left.0,004\left(U R_{\min }-45\right)\right]\left(\frac{h}{3}\right)^{0,3}\right\},\left\{K_{c b}+0,05\right\}\right)
\end{aligned}
$$

onde: max indica que a escolha deve recair no maior valor, $\mathrm{UR}_{\text {min }}$ é a média diária da umidade relativa do ar mínima e h é a altura da cultura, que era coletada em campo a cada 15 dias, na mesma semana da passagem do TM - Landsat 5.

$\mathrm{O}$ valor de $\mathrm{K}_{\mathrm{cb}}$ foi calculado para cada dia por meio da seguinte equação (Allen et al., 1998):

$$
K_{c b}=K_{c b(T a b)}+\left[0,04\left(u_{2}-2\right)-0,004\left(U R_{\min }-45\right)\right]\left(\frac{h}{3}\right)^{0,3}
$$

em que: $\mathrm{K}_{\mathrm{cb}(\mathrm{Tab})}$ é o valor do $\mathrm{K}_{\mathrm{cb}}$ da média e última fase de desenvolvimento da cultura apresentado para diferentes cultura na Tabela 17 do boletim FAO-56 (Allen et al., 1998). Os demais termos já definidos na Equação 14.

\section{RESULTADOS E DISCUSSÃO}

Os estádios fenológicos da cultura do algodão herbáceo (cultivar BRS 187 8H) sobre os quais foram obtidos os dados da razão de Bowen utilizados na validação do método aplicado estão apresentados na Tabela 1. A caracterização dos estádios foi elaborada a partir de observações de campo com base na metodologia proposta no boletim FAO-56 (Allen et al., 1998), a saber: estágio inicial - da emergência até a cultura propiciar $10 \%$ de cobertura do solo; desenvolvimento - dos 10\% de cobertura do solo até o início da floração; médio - início da floração até início da maturação; final - início da maturação até abertura total dos capulhos. A duração total do ciclo da cultivar foi de 105 dias, similar ao ciclo de outras cultivares, a exemplo da BRS - Marrom (Bezerra, 2007).

Os valores do Kc da cultura do algodão herbáceo obtidos em função do NDVI, denominados de Kc(NDVI), e os respectivos valores obtidos pela razão entre a ET estimada a partir da razão de Bowen e a ET0 estimada pelo método Penman-Monteith-FAO 56 (Allen et al., 1998), nomeados de Kc(razão de Bowen), são apresentados na Tabela 2.

Ainda na tabela 2 estão apresentadas para cada dia de aquisição das imagens, as partições dos respectivos valores do $\mathrm{K}_{\mathrm{c}}(\mathrm{NDVI})$. Observa-se que $\mathrm{K}_{\mathrm{e}}$ e $\mathrm{K}_{\mathrm{cb}}$ variaram de forma inversa entre as datas estudadas. A variabilidade tanto do Kcb como do $\mathrm{Ke}$, é diretamente influenciada pela cobertura do solo (fc) e pela altura média das plantas, ou seja, enquanto o Kcn aumenta a medida que planta cresce, e conseqüentemente propicia maior cobertura do solo, o Ke apresenta comportamento inverso, pois representa a evaporação do solo exposto que é sucessivamente menor a medida que a cultura se desenvolve. Assim, os valores do $\mathrm{K}_{\mathrm{e}}$ sucessivamente decrescentes, e os valores do $\mathrm{K}_{\mathrm{cb}}$ sucessivamente crescentes de uma data para outra, estão em consonância com fc, com a altura média das plantas e com NDVI da cultura nas referidas datas apresentados no gráfico da figura 3.

Observa-se ainda na tabela 2 que os resultados do Kc obtidos por ambas metodologias foram bastantes concordantes, embora os resultados obtidos por ambas as metodologias nos dias 17/11/2008 e 19/12/2009, quando a cultura se encontra em pleno estádio fenológico médio, são ligeiramente inferiores aos resultados constantes na literatura, que varia de 1,15 a 1,20 para o referido estádio (Doorenbos e Kassam, 1979; Allen et al., 1998). Farahani et al. (2008) também obtiveram resultados do Kc, do estádio fenológico médio inferiores ao da literatura em três anos de observação na parte oriental do Mediterrâneo, região setentrional da Síria. No citado estudo foram observado valores de 1,05, 1,05 e 1,04 nos anos de 2004, 2005 e 2006, respectivamente. Por sua vez, no dia 01/11/2008 os resultados do Kc obtido por ambas as metodologias, foram compatíveis com os resultados do estádio fenológico médio. Nesta data, a cultura se encontrava em pleno estágio de desenvolvimento vegetativo, mais precisamente a onze dias do seu final, e de

Tabela 1 - Divisão dos estádio fenológicos da cultura do algodão cultivado no municíío de Apodi-RN.

\begin{tabular}{lccc}
\hline \multicolumn{1}{c}{ ESTAGIO } & INÍCIO & FINAL & DURAÇÃO \\
\hline Inicial & $29 / 09 / 2008$ & $14 / 10 / 2008$ & 15 dias \\
Desenvolvimento & $15 / 10 / 2008$ & $11 / 11 / 2008$ & 29 dias \\
Médio & $12 / 11 / 2008$ & $19 / 12 / 2008$ & 38 dias \\
Final & $20 / 12 / 2008$ & $12 / 01 / 2009$ & 24 dias \\
\hline
\end{tabular}


Tabela 2 - Valores do Kc, Kcb e Ke estimados em função do NDVI obtido a partir das imagens TM - Landsat 5 e do Kc calculado a partir da ET estimada pelo método da razão de Bowen e pela ET0 estimada pelo método de Penman-Monteith-FAO-56 nas datas de aquisição das imagens.

\begin{tabular}{ccccccc}
\hline $\begin{array}{c}\text { Data de } \\
\text { aquisição } \\
\text { das } \\
\text { imagens }\end{array}$ & $\mathbf{K}_{\mathrm{c}}$ (NDVI) & $\mathbf{K}_{\mathrm{cb}}$ (NDVI) & $\mathbf{K}_{\mathrm{e}}$ (NDVI) & $\mathbf{K}_{\mathrm{e}, \max }$ & $\mathbf{K}_{\mathrm{e}}(\mathbf{N D V I}) / \mathbf{K}_{\mathrm{e} \max }$ & $\begin{array}{c}\mathbf{K}_{\mathrm{c}} \text { (Razão } \\
\mathbf{d e} \text { Bowen) }\end{array}$ \\
\hline $01 / 11 / 2008$ & 1,01 & 0,36 & 0,65 & 0,88 & 0,74 & 1,09 \\
$17 / 11 / 2008$ & 0,98 & 0,65 & 0,33 & 0,64 & 0,52 & 0,90 \\
$19 / 12 / 2008$ & 1,09 & 0,91 & 0,18 & 0,49 & 0,37 & 1,08 \\
\hline
\end{tabular}

acordo com Doorenbos e Kassam (1979) e Allen et al. (1998), os valores característicos do algodoeiro para esse período varia 0,35 , no início do estádio, a aproximadamente 1,2 no final. Vale lembrar que o valor inicial é bastante variável, pois é fortemente influenciado pela freqüência e intensidade de eventos como irrigação e precipitação (Allen et al., 1998). No entanto, a compatibilidade dos resultados do Kc no dia 01/11/2008, estágio de desenvolvimento vegetativo, com os valores observados nas datas no estádio fenológico médio, 17/11/2008 e 19/12/2008, foram atribuídos a um incremento nos valores da ET devido à alta taxa de evaporação, oriunda da freqüente irrigação uma vez que nessa data foi aplicada uma lâmina de $20 \mathrm{~mm}$. Segundo López-Urrea et al. (2009a), a componente evaporação do solo presente na ET, apresenta valores consideravelmente altos quando um sistema de irrigação por aspersão é usado com freqüência, principalmente nos estágios fenológicos iniciais quando o solo ainda apresenta considerada fração exposta.

Uma forte e contundente evidência da elevada evaporação do solo do dia 01/11/2008, confirmando a afirmação de López-

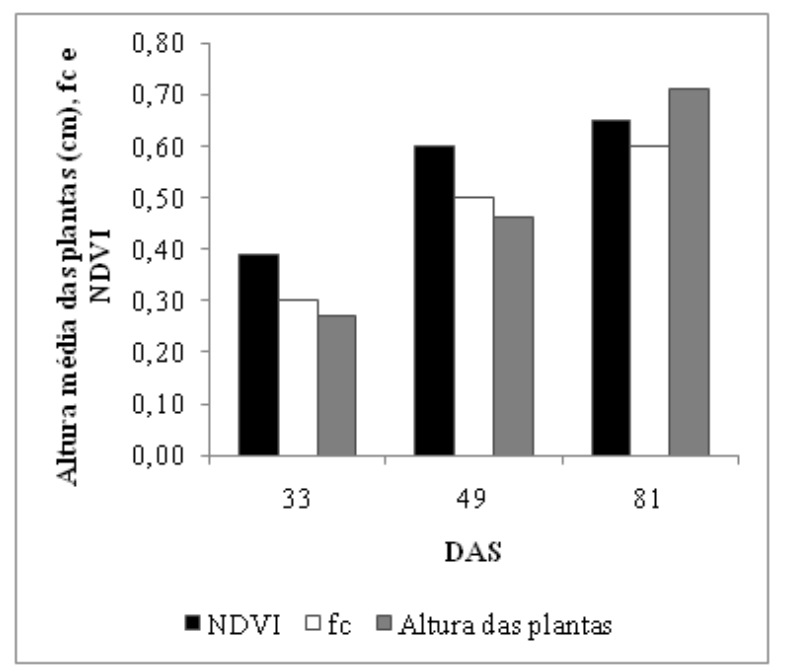

Figura 3 - Altura, fração de cobertura e NDVI da cultura do algodão no dia da aquisição das imagens TM - Landsat 5
Urrea et al. (2009a), é a razão $\mathrm{K}_{\mathrm{e}}(\mathrm{NDVI}) / \mathrm{K}_{\mathrm{e}, \max }$ (Tabela 2) indicando que a evaporação deste dia correspondeu a $74 \%$ do valor máximo, a mais elevada entre todos os dias estudados. A componente evaporação presente na ET atinge o seu valor máximo, quando a superfície do solo está completamente úmida por ocasião de precipitação ou irrigação, e diminui à medida que a umidade dessa superfície diminui (Allen et al., 1998; Er-Raki et al., 2007).

Na figura 4 é apresentada a curva do Kc(Razão de Bowen) para todo o ciclo da cultura. Devido à influência da umidade da superfície do solo sobre a ET, oriunda da freqüente irrigação, os valores do Kc são reportados como média de cinco dias para que uma função polinomial pudesse ser ajustada, conforme sugerem López-Urrea et al. (2009a). A função polinomial de segundo grau tem sido ajustada em estudos desenvolvidos para diferentes culturas e tem propiciado bons desempenhos apresentando altos coeficientes de correlação e determinação (López-Urrea et al., 2009a, b), para as culturas do trigo e brócolis. No entanto, no presente estudo o melhor ajuste foi alcançado por uma função polinomial de terceiro grau, apresentando coeficiente de determinação superior a 0,80, coeficiente de correlação de aproximadamente 0,90 . Dessa forma, fica evidente que os valores médios de cinco dias do Kc dos estágios fenológicos inicial e de desenvolvimento (5 a 45 DAE), com base nas observações de campo e na curva da função ajustada apresentados na figura 3, são mais próximos dos seus respectivos valores constantes na literatura, já citados anteriormente, para os referidos estádios. Ainda na Figura 4, na comparação entre a curva dos valores médios e a curva da função ajustada, pode-se verificar que o resultado diário do Kc obtido a partir do NDVI no dia 01/11/2008 é consideravelmente superior aos valores médios de cinco dias. Nas duas outras datas, 17/11 e 19/12, a exemplo da comparação com os resultados diários da Tabela 2, também são observadas boas concordâncias com os valores médios. Assim, os elevados valores do Kc apresentado em 01/11, não correspondem à falhas, limitações ou erros associados aos respectivos métodos, mas sim como 
demonstrado, devido a sensibilidade de ambas as metodologias quanto a uma característica intrínseca do sistema de irrigação por aspersão. López-Urrea et al. (2009a) também verificaram o mesmo comportamento no Kc do trigo, utilizando valores da ET obtidos através de medidas lisimétricas.

Ainda na figura 4 , as barras verticais representam o desvio padrão associado a cada media dos cinco dias. Observa-se que os desvios padrão foram mais intensos e estão associados às médias do início até os $40 \mathrm{DAE}$, evidenciando o efeito da irrigação na ET da cultura que sofreu constantes oscilações em seus valores entre dias sem e com irrigação. Essas oscilações estão evidenciadas em igual período no ciclo em igual período na figura 5 .

A ET diária obtida com base no $\mathrm{K}_{\mathrm{c}}(\mathrm{NDVI})$ apresentou grande concordância quando comparada com os valores de ET estimados em campo baseadas na técnica de razão de Bowen, conforme apresentado na figura 5 e nos dados da Tabela 3. As precisões alcançadas pelas estimativas da ET através do $K_{c}(N D V I) \cdot E T_{0}$, são semelhantes e comparáveis aos desempenhos alcançados por algoritmos que utilizam técnicas de sensoriamento remoto e que são constituídos de metodologias de processamento bastante robustas e complexas a exemplo do SEBAL (Bezerra et al., 2008a; Li et al., 2008), S-SEBI (Bezerra et al., 2008b) e METRIC (Allen et al., 2007). Segundo Bastiaanssen et al. (1998), diferenças nas magnitudes da Tabela 3 se enquadram dentro da faixa das imprecisões instrumentais.

A ET sazonal da cultura do algodoeiro foi obtida para os períodos com disponibilidade de imagens livres da presença de nuvens. Assim, só foi possível a obtenção da ET

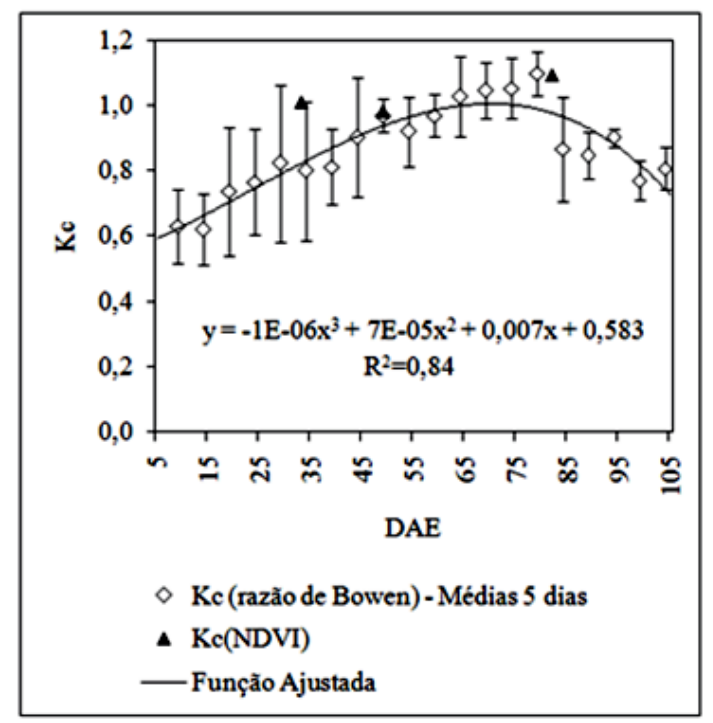

Figura 4 - Ajuste do Kc obtido a partir da ET estimada pelo método da razão de Bowen e da ET0 estimada pelo método Penman-MonteithFAO-56 e os valores da Kc obtido pelas imagens orbitais através do NDVI. do estágio fenológico médio, isto é, de 12/11 a 19/12, quando foram obtidas duas imagens - 17/11/2008 e 19/12/2008. Outro fator favorável para obtenção da ET do referido período é o comportamento da curva característica do respectivo $\mathrm{K}_{\mathrm{c}}$, que é aproximadamente constante, segundo Allen et al. (1998). Ainda segundo os mesmos autores, o $\mathrm{K}_{\mathrm{c}}$ dos estádios fenológicos inicial e desenvolvimento, apresentam variabilidade diária com comportamento ascendente, enquanto que o $\mathrm{K}_{\mathrm{c}}$ corresponde ao último estágio, mostra uma variabilidade segundo uma curva descendente, conforme Allen et al. (1998). Dessa forma, são necessárias medições do NDVI com grande freqüência temporal. $\mathrm{O}$ valor atípico do $\mathrm{K}_{\mathrm{c}}$ obtido a partir dos dados da única imagem obtida durante o primeiro e segundo estádios (01/11/2008), por razões já antes discutidas e a indisponibilidade de imagens livres da presença de nuvens durante o último estádios, inviabilizou o cálculo da ET sazonal correspondente aos respectivos períodos. No entanto, o cálculo da ET do estádio médio é considerado relevante, pois constitui a fase de maior consumo hídrico, demonstrado na Tabela 4, onde se verifica que o consumo hídrico do referido estádio corresponde a aproximadamente $43 \%$ do total.

Os resultados da ET sazonal da cultura obtidos com base na combinação $\mathrm{K}_{\mathrm{c}}(\mathrm{NDVI})$ e as respectivas estimativas baseadas em observações de campo estão apresentados na Tabela 5. O bom desempenho alcançado pela metodologia utilizada na obtenção da ET sazonal do período correspondente ao estádio fenológico médio da cultura, corrobora com outros estudos realizados em diferentes culturas. Simmoneaux et al. (2008)

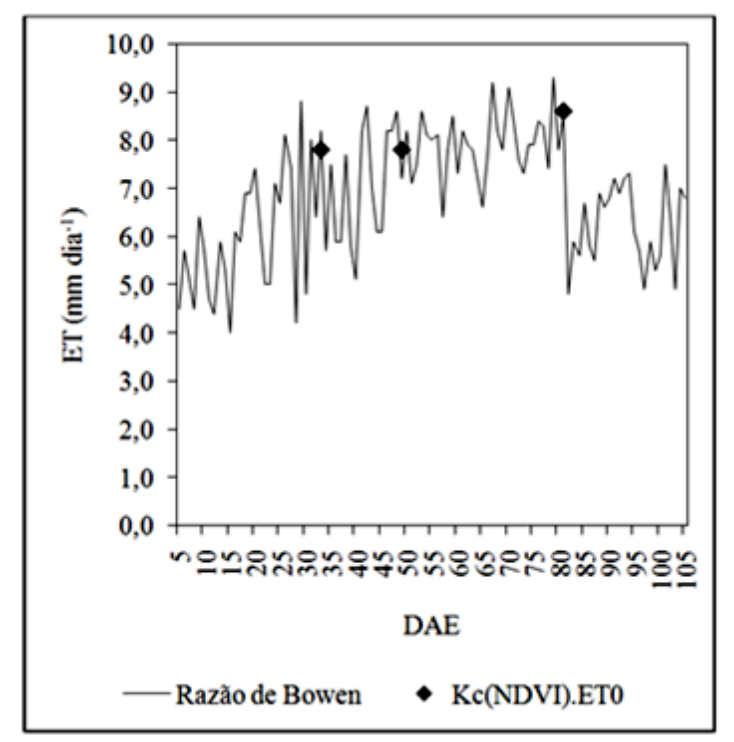

Figura 5 - Evolução do temporal da ET da cultura do algodão e os valores da ET obtida a partir do Kc calculado pela metodologia avaliada. 
Tabela 3 - Comparação dos valores diários da ET da cultura do algodão obtidos a partir da metodologia avaliada com valores estimados através do método da razão de Bowen, nas datas de aquisição das imagens orbitais.

\begin{tabular}{lcclccc}
\hline & \multicolumn{2}{c}{$\mathrm{ET}\left(\mathrm{mm} \mathrm{dia}{ }^{-1}\right)$} & & \multicolumn{2}{c}{ Diferença } \\
\cline { 2 - 3 } \cline { 5 - 6 } & Razão de Bowen & $K_{0}($ NDVI $) \cdot E T_{0}$ & & $\mathrm{~mm}$ & $(\%)$ \\
\hline $\mathbf{1 / 1 1 / 2 0 0 8}$ & 8,2 & 7,8 & & 0,4 & 4,9 \\
$\mathbf{1 7 / 1 1 / 2 0 0 8}$ & 7,2 & 7,8 & & $-0,6$ & 8,3 \\
$\mathbf{1 9 / 1 2 / 2 0 0 8}$ & 8,5 & 8,6 & & $-0,1$ & 1,2 \\
Média & 8,0 & 8,1 & & $-0,1$ & 1,3 \\
\hline
\end{tabular}

Tabela 4 - Consumo hídrico de cada estádio fenológico e total da cultura do algodão estimados pela técnica de razão de Bowen e as respectivas porções consumidas em cada estádio.

\begin{tabular}{lcc}
\hline \multicolumn{1}{c}{ Estádio fenológico } & $\mathrm{ET}(\mathrm{mm})$ & $\mathrm{ET}_{\text {estidio }} / \mathrm{ET}_{\text {total }}$ \\
\hline Inicial & 56,2 & 0,09 \\
Desenvolvimento & 186,6 & 0,27 \\
Médio & 304,2 & 0,43 \\
Final & 148,4 & 0,21 \\
Total & 695,4 & - \\
\hline
\end{tabular}

Tabela 5 - Resultados da ET sazonal do estágio fenológico médio e dos meses de novembro e dezembro de 2008 separadamente, obtidos a partir do $\mathrm{Kc}(\mathrm{NDVI})$

\begin{tabular}{|c|c|c|c|}
\hline & $\begin{array}{l}\text { ET (mm) Estádio } \\
\text { Fenológico Médio } \\
(12 / \text { nov - } 18 / \mathrm{dez})\end{array}$ & $\begin{array}{l}\text { ET }(\mathrm{mm}) \\
\text { Novembro }\end{array}$ & $\begin{array}{l}\text { ET }(\mathrm{mm}) \\
\text { Dezembro }\end{array}$ \\
\hline Razão de Bowen (mm) & 297,2 & 231,1 & 228,6 \\
\hline $\mathrm{K}_{\mathrm{c}}(\mathrm{NDVI}) \cdot \mathrm{ET}_{0}(\mathrm{~mm})$ & 302,2 & 244,6 & 254,5 \\
\hline$\left|\mathrm{ET}_{\text {RazäoEowen }}-\mathrm{ET}_{\mathrm{Kc}(\mathrm{NDVI})}\right|(\mathrm{mm})$ & 5,0 & 13,5 & 25,9 \\
\hline$\left|\mathrm{ET}_{\mathrm{Razä \circ Bowen}}-\mathrm{ET}_{\mathrm{Kc}(\mathrm{NDVI})}\right| / \mathbf{n}^{*}\left(\mathrm{~mm} \mathrm{dia}^{-1}\right)$ & 0,1 & 0,5 & 0,8 \\
\hline Diferença (\%) & 1,7 & 5,8 & 11,3 \\
\hline
\end{tabular}

"número de dias do período correspondente.

observaram que para o período que dispunha de observações, cerca de 160 dias, considerando três locais de observações em pomares de citros, damasqueiros e videiras a estimativa da ET média diária foi de $3,48 \mathrm{~mm}$, o que significa uma diferença de apenas $4,8 \%$ em comparação com as medições. Hunsaker et al. (2005a) constataram que a estimativa da ET sazonal do trigo, nas safras 1996 e 1997, diferenciaram em 33 mm (5\%) e 18 $\mathrm{mm}(3 \%)$, respectivamente, dos resultados estimados através do método do balanço de água no solo. No entanto, ainda no mesmo estudo citado anteriormente, a média da diferença percentual absoluta entre os resultados diários estimados e medidos variou entre de $9 \%$ e $10 \%$.

No entanto, vale lembrar que o bom desempenho alcançado pelo método no estágio fenológico médio, também está associado ao fato do $\mathrm{K}_{\mathrm{c}}$ do referido estágio ser praticamente constante e a utilização de duas imagens, no início e fim do 
Tabela 6 - Resultados da ET sazonal do período de 01 novembro e 31 dezembro de 2008 obtidos a partir do Kc(NDVI)

01/Nov - 31/Dez de 2008

\begin{tabular}{|c|c|}
\hline Razão de Bowen (mm) & 459,7 \\
\hline $\mathrm{K}_{\mathrm{c}}(\mathrm{NDVI}) \cdot \mathrm{ET}_{0}(\mathrm{~mm})$ & 499,1 \\
\hline$\left|\mathrm{ET}_{\mathrm{RazäoBowen}}-\mathrm{ET}_{\mathrm{Kc}(\mathrm{NDV})}\right|(\mathrm{mm})$ & 39,4 \\
\hline$\left|\mathrm{ET}_{\text {RazäoBowen }}-\mathrm{ET}_{\mathrm{Kc}(\mathrm{NDV})}\right| / \mathbf{n}{ }^{*}\left(\mathrm{~mm} \mathrm{dia}^{-1}\right)$ & 0,6 \\
\hline Diferença (\%) & 8,6 \\
\hline
\end{tabular}

*número de dias do período correspondente.

estágio, contribuiu de forma positiva para a precisão alcançada pelo método no presente trabalho.

Uma evidência de que a precisão da técnica está diretamente ligada a disponibilidade das medidas do NDVI em alta freqüência temporal, principalmente nos estágios fenológicos iniciais, é expressa na Tabela 5, onde estão apresentadas as estimativas da ET da cultura do algodão dos meses de novembro e dezembro de 2008. O resultado mais modesto do mês de dezembro é atribuído a escassez de medidas do NDVI, uma vez que foram utilizados apenas dados de uma única imagem de satélite - 19/12/2008 e mesmo assim obtida no último dia do estágio fenológico médio. Assim, foi verificada uma diferença de $25,9 \mathrm{~mm}$ entre os resultados estimados e medidos durante o mês, o que implicou numa diferença de $11,3 \%$. Já no mês de novembro foram utilizadas medições do NDVI oriundas de duas imagens, 01 e 17 de novembro de 2008, onde se constata que todas as diferenças analisadas representam praticamente a metade das mesmas analisadas em dezembro. Porém, na análise sobre todo o período, isto é, novembro mais dezembro, cujos dados estão apresentados na Tabela 6 , verifica-se que a diferença de $8,6 \%$ pode ser considerada confiável, o que representa diferenças médias diárias de apenas $0,6 \mathrm{~mm} \mathrm{dia}^{-1}$. Todos os resultados estão em conformidade com o desempenho do método alcançado em outros estudos, a exemplo de Hunsaker et al. (2007a), que estimaram a ET do algodão submetidas a tratamentos nutricionais diferenciados no estado do Arizona.

Nesse contexto, a dependência de dados de imagens de satélites representa uma limitação relevante na aplicação da técnica, uma vez que somente imagens livres da presença de nuvens podem ser utilizadas. Mas, por outro lado, a utilização das refletâncias espectrais obtidas em campo viabiliza o emprego da técnica, conforme (Hunsaker et al., 2003; 2005a, b; 2007a, b) face à praticidade, simplicidade e acurácia da mesma.

\section{CONCLUSÕES}

Diante dos resultados conclui-se que:

A metodologia avaliada propiciou resultados diários e sazonais da ET do algodoeiro com nível de precisão satisfatório e aceitável, apresentando diferenças inferiores a 10\%, quando comparadas com estimativas da técnica de razão de Bowen.

O desempenho da metodologia avaliada é compatível com o desempenho de algoritmos que utilizam dados de sensoriamento remoto (principalmente imagens de satélites) e que são constituídos de metodologias bastante complexas, a exemplo do SEBAL e do S-SEBI.

\section{AGRADECIMENTOS}

Os autores agradecem ao CNPq/CT-HIDRO pela concessão de bolsa de doutorado do primeiro autor, ao CNPq pela concessão de bolsa de Produtividade em Pesquisa ao segundo autor e pelo financiamento do Projeto Geração e Transferência de Tecnologias para a Sustentabilidade do Algodoeiro no Semiárido Nordestino, convênio ATECEL-FINEP-EMBRAPA, $\mathrm{n}^{\circ}$. 591-07 e ao INMET pela disponibilidade dos dados meteorológicos utilizados neste estudo.

\section{REFERÊNCIAS BIBLIOGRÁFICAS}

ALLEN, R. G. Using the FAO-56 dual crop coefficient method over an irrigated region as part of an evapotranspiration intercomparison study. Journal of Hydrology, Amsterdam, v. 229, p. 27-41, 2000.

ALlEN, R. G.; PEREIRA, L. S.; RAES, D.; SMITH, M. FAO Irrigation and Drainage Paper $\mathrm{N}^{\circ}$ 56. Crop Evapotranspiration (guidelines for computation crop water requirements, Rome: FAO, 1998. 
ALLEN, R. G.; TASUMI, M.; MORSE, A.; TREZZA, R.; WRIGHT, J. L.; BASTIAANSSEN, W.; KRAMBER, W.; LORITE, I.; ROBINSON, C. Satellite-based energy balance for mapping evapotranspiration with internalized calibration (METRIC) - Applications. Journal of Irrigation and Drainage Engineering, Reston, v. 133, n. 4, p. 395-406, 2007.

BASTIAANSSEN, W. G. M.; PELGRUN, H.; WANG, J.; MA, Y.; MORENO, J. F.; ROERINK, G. J.; VAN DER WAL, T. A remote sensing surface energy balance algorithm for land (SEBAL).: Part 2: Validation. Journal of Hydrology, Amsterdam, v. 212-213, p. 213-229, 1998.

BAUSCH, W. C.; NEALE, C. M. U. Spectral inputs improve corn crop coefficients and irrigation scheduling. Transactions of ASAE, St. Joseph, v. 32, n. 6, p. 1901-1908, 1987.

BEZERRA, B. G.; SILVA, B. B.; FERREIRA, N. J. Estimativa da evapotranspiração real diária utilizando-se de imagens digitais TM - Landsat 5. Revista Brasileira de Meteorologia, São José dos Campos, v. 23, n. 3, p. 305-317, 2008 a.

BEZERRA, B. G.; SILVA, B. B.; FERREIRA, R. C.; BORGES, V. P. Estimativa da evapotranspiração diária baseada em dados de sensoriamento remoto. In.: Workshop Inovações Tecnológicas na Irrigação, 2, \& Simpósio Brasileiro sobre o uso Múltiplo da Água, 1. Anais... Fortaleza: Inovagri, 2008b. 1 CD-ROM.

BEZERRA, J. R. C. Crescimento, Desenvolvimento e Rentabilidade do Algodoeiro BRS 200 - Marrom Irrigado. 2007. 88f. Tese (Doutorado em Recursos Naturais) Universidade Federal de Campina Grande, Campina Grande, 2007.

CHANDER, G.; MARKHAM, B. Revised Landsat-5 TM Radiometric Calibration Procedures and Postcalibration Dynamic Ranges. IEEE Transactions on Geoscience and Remote Sensing. Piscataway, v. 41. n. 11, p. 2674-2677, 2003. CHOUDhuRY, B. J.; AHMED, N. U.; IDSO, S. B.; REGINATO, R. J.; DAUGHTRY, C. S. T. Relations between evaporation coefficients and vegetation indices studies by model simulations. Remote Sensing Environment, New York, v. 50, p. 1-17, 1994.

DOORENBOS, J.; KASSAM, A. H. Yields response to water. (FAO: Irrigation and Drainage Paper, 33), Rome: FAO, 1979.

DUCHEMIN, B.; HADRIA, R.; ER-RAKI, S.; BOULET, G.; MAISONGRANDE, P.; CHEHBOUNI, A.; ESCADAFAL, R.; EZZAHAR, J.; HOEDJES, J. C. B.; KHARROU, M. H.; KHABBA, S.; MOUGENOT, B.; OLIOSO, A.; RODRIGUEZ, J. -C.; SIMONNEAUX, V. Monitoring wheat phenology and irrigation in Central Morocco: On the use of relationships between evapotranspiration, crops coefficients, leaf area index and remotely-sensed vegetation indices. Agricultural Water Management, Amsterdam, v. 79, p. 1-27, 2006.
ER-RAKI, S.; CHEHBOUNI, A.; GUEMOURIA, N.; DECHEMIN, B.; EZZAHAR, J.; HADRIA, R. Combining FAO-56 model and ground-based remote sensing to estimate water consumptions of wheat crops in a semiarid region. Agricultural Water Management, Amsterdam, v. 87, p. 4154, 2007.

FARAHANI, H. J.; OWEIS, T. Y.; IZZI, G. Crop coefficient for drip-irrigated cotton in a Mediterranean environment. Irrigation Science, New York, v.26, p.375-383, 2008.

HUNSAKER, D. J.; PINTER JR., P. J.; KIMBALL, B. A. Wheat basal crop coefficients determined by normalized difference vegetation index. Irrigation Science, New York, v. 24, p. 1-14, 2005a.

HUNSAKER, D. J.; BARNES, E. M.; CLARKE, T. R.; FITZGERALD, G. J.; PRINTER JR., P. J. Cotton irrigation scheduling using remotely sensed and FAO-56 basal crop coefficients. Transactions of the ASAE, St. Joseph, v. 48, n. 4, p. 1395-1407, 2005b.

HUNSAKER, D. J.; FITZGERALD. G. J.; FRENCH, A. N.; CLARKE, T. R.; OTTMAN, M. J.; PRINTER JR., P. J. Wheat irrigation management using multispectral crop coefficients: I. Crop evapotranspiration prediction. Transactions of the ASABE, St. Joseph, v. 50, n. 6, p. $2017-$ 2033, 2007a.

HUNSAKER, D. J.; FITZGERALD, G. J.; FRENCH, A. N.; CLARKE, T. R.; OTTMAN, M. J.; PRINTER JR., P. J. Wheat irrigation management using multispectral crop coefficients: II. Irrigation scheduling performance, grain yield, and water use efficiency. Transactions of the ASABE, St. Joseph, v. 50, n. 6, p. 2035-2050, 2007 b.

HUNSAKER, D. J.; PRINTER JR., P. J.; BARNES, E. M.; KIMBALL, B. A. Estimating cotton evapotranspiration crop coefficients with a multispectral vegetation index. Irrigation Science, New York, v. 22, p. 95-104, 2003.

JACKSON, R. D.; IDSON, S. B. REGINATO, R. J.; PRINTER JR., P. J. Remotely sensed crop temperatures and reflectances as inputs to irrigation scheduling. In: Proceedings of the Special Conference on Irrigation and Drainage, Boise, Idaho. ASCE, New York, p. 390-397, 1980.

LI, H.; ZHENG, L.; LEI, Y.; LI, C.; LIU, Z.; ZHANG, S. Estimation of water consumption and crop water productivity of winter in the North China Plain using remote sensing technology. Agricultural Water Management, Amsterdam, v. 95, p. 1271-1278, 2008.

LÓPEZ-URREA, R.; MONTORO, A.; GONZÁLEZPIQUERAS, J.; LÓPEZ-FUSTER, P.; FERERES, E. Water use of spring wheat to rise water productivity. Agricultural Water Management, Amsterdam, v. 96, p. 1305-1310, 2009a.

LÓPEZ-URREA, R.; MONTORO, A.; LÓPEZ-FUSTER, P.; FERERES, E. Evapotranspiration and responses to irrigation 
of broccoli. Agricultural Water Management, Amsterdam, v. 96, p. 1155-1161, 2009b.

MARKHAM, B. L. \& BAKER, J. L. Landsat MSS and TM PostCalibration Dynamic Rangers, Exoatmospheric Reflectance and At-Satellite Temperatures. EOSAT Landsat Tech, 1986.

MORAN, M. S.; MASS, S. J.; PINTER JR., P. J. Combining remote sensing and modelling for estimating surface evaporation and biomass production. Remote Sensing Review, London, n. 12, p. 335-353, 1995.

NEALE, C. M. U.; BAUSCH, W. C., HEERMAN, D. F.; Development of reflectance-based crop coefficients for corn. Transaction of ASAE, St. Joseph, v. 32, n. 6, p. 1891-1899, 1989.

PEREZ, P. J.; CASTELVI, F.; IBAÑEZ, M.; ROSSEL, J. I. Assessment of reliability of Bowen ratio method for partitioning fluxes. Agricultural and Forest Meteorology, Amsterdam, v. 97, p. 141-150, 1999.
ROUSE, J. W.; HAAS, R. H.; SCHELL, J. A.; DEERING, D. W. HARLAN, J. C. Monitoring the vernal advancement and retro gradation of natural vegetation. NASA/GSFC, Type III, Final report, Greenbelt, MD, p. 1-371, 1974.

SIMONNEAUX, V.; DUCHEMIN, B.; HELSON, D.; ERRAKI, S.; OLIOSO, A.; CHEHBOUNI. A. G. The use of high-resolution image time series for crop classification and evapotranspiration estimate over an irrigated are in central Morocco. International Journal of Remote Sensing, Abingdom, v. 29, n. 1, p. 95-116, 2008.

THORNTHWAITE, C.W. An approach toward a rational classification of climate. Geographical Review, New York, v. 38, p. 55-94, 1948. 